\title{
IMPORTÂNCIA DO PLANEJAMENTO AMOSTRAL EM ESTUDOS ECOLÓGICOS: UM ESTUDO DE CASO NO LITORAL DO PARANÁ
}

\author{
Sample design importance in ecological studies: \\ a case study on Paraná coast
}

Fabiana Cézar Félix ${ }^{l}$

Carlos Werner Hackradt ${ }^{2}$

\section{Resumo}

Muitos estudos ecológicos são conduzidos sem a prévia existência de delineamentos amostrais adequados, de forma que dados são coletados na ausência de critérios e com finalidades não evidentes, e somente após sua coleta julgam-se quais análises estatísticas são as mais apropriadas aos dados recolhidos. Com base em dois estudos realizados com peixes no litoral do Paraná, mostra-se como a falta de replicação pode levar a conclusões errôneas acerca da influência dos fatores analisados sobre a comunidade amostrada. Anecessidade de bom planejamento amostral e a determinação prévia das análises estatísticas aplicáveis aos dados a serem coletados são essenciais para se obter respostas adequadas à(s) hipótese(s) para o qual foram delineadas.

Palavras-chave: Desenho amostral; Análise de variância; Pseudoreplicação.

\section{Abstract}

Many ecological studies are conducted without appropriate sampling design, data collection are made without criteria and non evident purposes and only after its collection it is judged which statistical analysis are more appropriate to gathered data. Based on two fish studies realized at Paraná coast, it displays how lack of replication could lead to wrong conclusions about factors' influence under sampled community. The necessity of well designed sampling program and previous determination of statistical analysis applicable to data is essential to obtain adequate answers to hypothesis for whose they were delineated.

Keywords: Sample design; Variance analysis; Pseudoreplication.

Bióloga, M. Sc. Instituto Nautilus de Pesquisa e Conservação da Biodiversidade. e-mail: felixfabiana@yahoo.com.br

2 Biólogo, M. Sc. Instituto Nautilus de Pesquisa e Conservação da Biodiversidade. e-mail: hackkradtcw@gmail.com 


\section{Introdução}

Todo estudo ecológico nasce de uma observação feita durante uma campanha de campo no qual o pesquisador, ao observar um determinado fenômeno ecológico ou um comportamento biológico, idealiza modelos que tentam explicá-los (1). A partir destes modelos, surgem as hipóteses de trabalho que serão postas à prova por meio da condução de experimentos em laboratório, ou in situ, mediante amostragem de uma população. Para cada hipótese estabelecida, constrói-se um desenho amostral específico para a sua solução e conseqüentemente uma análise de dados que vise a testar estatisticamente os dados coletados e/ou observados (2). Se a hipótese nula (igualdade entre os tratamentos) for aceita, resulta que o modelo utilizado não é capaz de explicar o fenômeno observado e, portanto, este deve ser descartado e um novo modelo construído. Contudo, se a hipótese nula é rejeitada, isso não significa que o modelo está absolutamente certo e sim que para aquelas situações em que foi testado este se aplica adequadamente (2). Por este motivo, é necessário que o modelo sofra contínuos testes, em níveis cada vez mais generalistas, a fim de contemplar todas as situações possíveis explicadas por este (3).

Infelizmente, este processo intelectual muitas vezes não chega a ocorrer e grande parte dos pesquisadores vai a campo sem um delineamento amostral preciso que se ocupe em responder perguntas de interesse científico específico. O que se observa em muitos trabalhos é a excessiva preocupação com séries temporais longas em detrimento das variações espaciais, que são esquecidas talvez por falta de logística, financiamento e/ou a soma destas, limitando o poder de interpretação das respostas encontradas $(4,5)$.
Este trabalho se propõe a fazer uma análise crítica sobre delineamento amostral e suas limitações durante o teste de hipóteses, utilizando-se para tal dois estudos de caso baseados em trabalhos realizados no litoral do Paraná entre os anos de 2004 a 2006.

\section{Material e métodos}

Dois estudos realizados com a ictiofauna de ambiente praial foram utilizados para comparar a eficiência e poder de seus respectivos delineamentos amostrais em responder às hipóteses de trabalho para qual foram desenhados. Para ambos os trabalhos, será analisada a relação entre a hipótese a ser testada e o planejamento amostral efetuado, procurando esclarecer suas limitações e erros. A fim de testar a força e eficiência de tais planejamentos, foram construídas tabelas do tipo "split-plot" (3), nas quais foram identificadas, em cada estudo, as fontes de variação, o grau de liberdade, as variâncias e a razão de F. Esta tem como objetivo avaliar quais são as fontes de variação que influenciam no resultado do estudo e avaliar a potência ou força do teste estatístico em responder às questões estabelecidas. Todos os fatores analisados (energia, contaminação e tempo) foram considerados fixos durante a construção da tabela, pois havia interesse em saber individualmente a influência de cada um dos fatores e de suas interações. Se não há interesse por parte do pesquisador em individualizar as influências dos fatores, este pode considerá-lo como aleatório (3).

Para o primeiro estudo, realizado em 3 praias arenosas (1A-3A) de Pontal do Paraná (Figura 1), procurou-se avaliar se a comunidade de peixes variava segundo o grau energético das zonas de arrebentação amostradas. Para tanto, foram formuladas as seguintes hipóteses: 


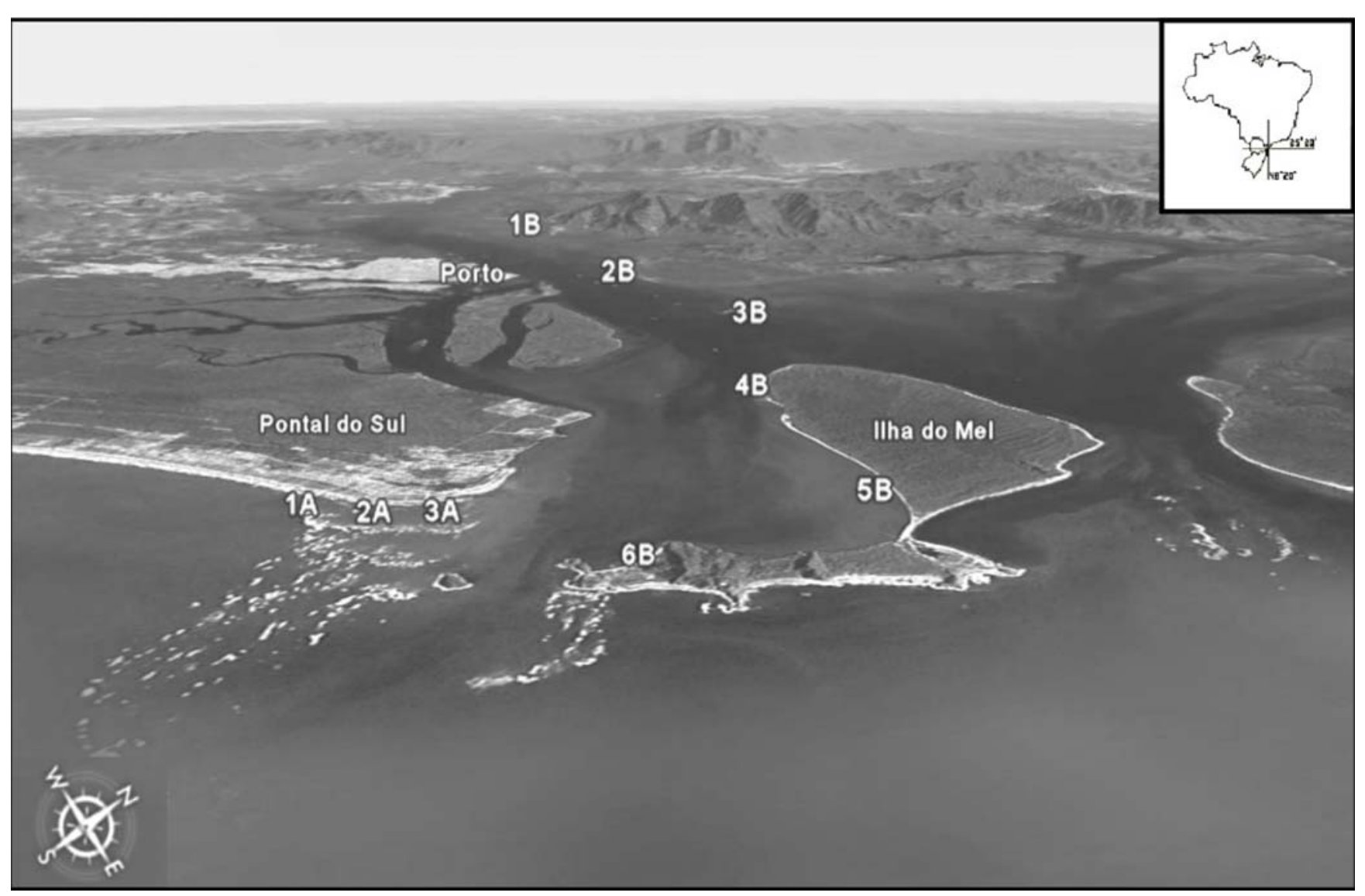

\section{Figura 1- Mapa mostrando a localização das praias do primeiro(1-3 A) e do segundo estudo(1-6 B), realizados em Pontal do Paraná, PR, Brasil}

Fonte: Google Earth.

1. $\mathrm{H}_{0}$ : os descritores da comunidade (número de indivíduos e espécies, biomassa, índice de diversidade de Shannon-Wiener e equitatividade de Pielou) são iguais nas três condições de energia.

$\mathrm{H}_{\mathrm{A}}$ : os descritores da comunidade decrescem com o aumento do gradiente de energia.

2. $\mathrm{H}_{0}$ : os descritores da comunidade são iguais entre os meses estudados.

$\mathrm{H}_{\mathrm{A}}$ : os descritores da comunidade variam sazonalmente ao longo do tempo.

O planejamento amostral incluía arrastos mensais durante o período de 1 ano utilizando-se de uma rede tipo picaré de $2,6 \mathrm{~m}$ de altura, $15 \mathrm{~m}$ de comprimento e $2 \mathrm{~m}$ de saco com malhagem de $0,5 \mathrm{~cm}$ em toda a extensão desta. Em cada uma das praias, que se situavam ao longo de um gradiente de exposição às ondas, 5 arrastos de $30 \mathrm{~m}$ de extensão cada e intervalos regulares de $5 \mathrm{~m}$ entre arrastos sucessivos foram conduzidos para a amostragem da ictiofauna (Figura 2a). 

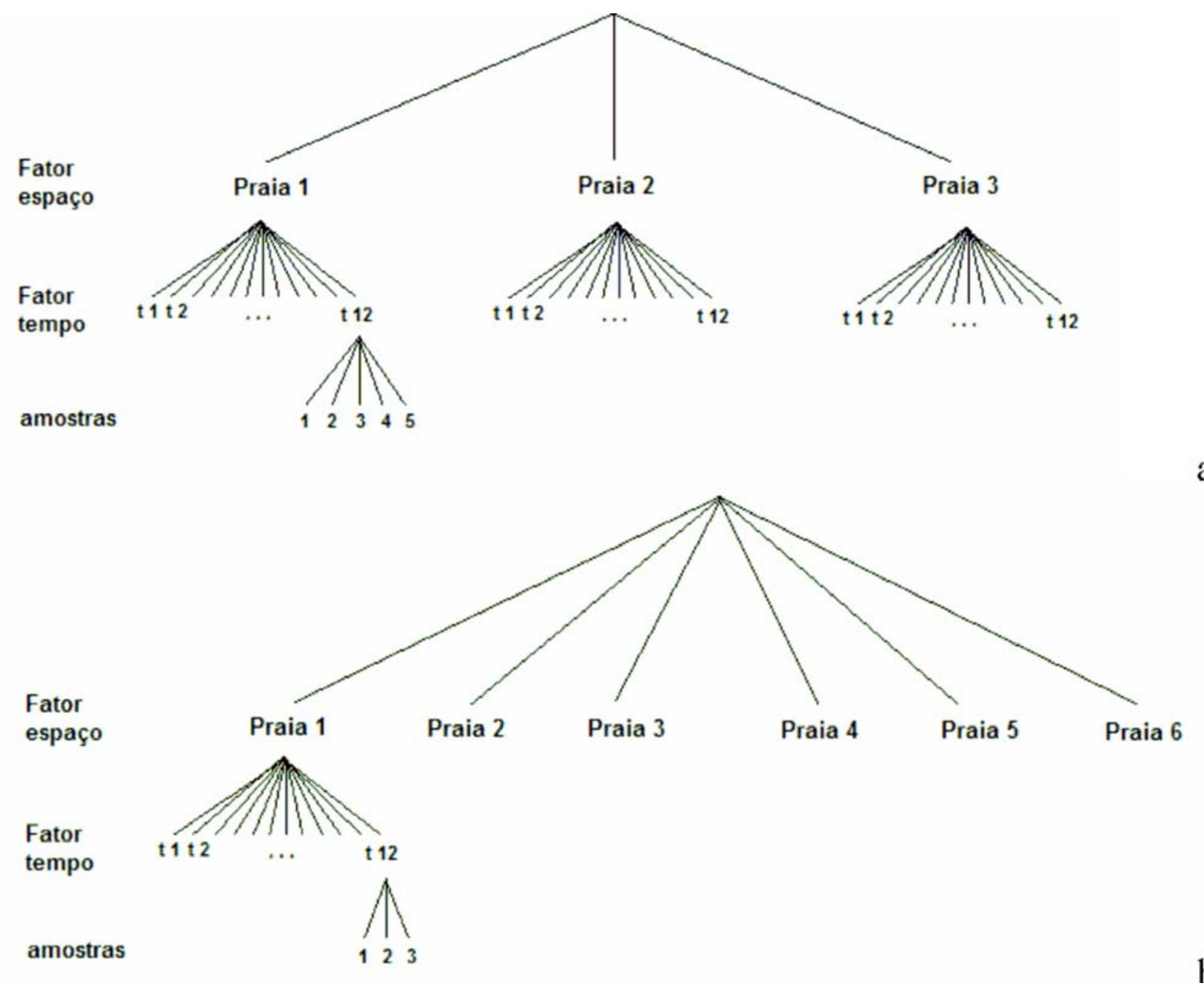

Figura 2- Desenhos amostrais esquemáticos evidenciando o erro cometido no estudo 1 (a) e no estudo 2 (b) ao considerar as praias como fatores de energia e contaminação, respectivamente e não comofatores espaciais

O segundo estudo preocupou-se em responder à seguinte pergunta: será que a explosão e vazamento de um navio petroquímico influenciam na composição e estrutura da ictiofauna de praias espaço-temporalmente? As seguintes hipóteses foram formuladas:

1. $\mathrm{H}_{0}$ : os descritores da comunidade são iguais nas 6 praias estudadas.

$\mathrm{H}_{\mathrm{A}}$ : os descritores da comunidade decrescem a partir da praia controle (mais interna) ao longo do gradiente de contaminação.

2. $\mathrm{H}_{0}$ : os descritores da comunidade são iguais entre os meses.

$\mathrm{H}_{\mathrm{A}}$ : os descritores da comunidade variam sazonalmente ao longo do tempo. 
Para respondê-las, foram escolhidas seis praias estuarinas inseridas no Complexo Estuarino Baía de Paranaguá (Figura 1), das quais uma se situava anterior ao porto (1B) e as demais seguiam um gradiente de diluição em direção à abertura da baía (2-6B). Três arrastos mensais de $20 \mathrm{~m}$ de extensão cada e intervalos regulares de $5 \mathrm{~m}$ entre arrastos sucessivos foram conduzidos em cada praia durante 12 meses, utilizando a mesma rede descrita acima para o outro estudo (Figura $2 b$ ).

\section{Resultados e discussão}

A análise das variâncias e da razão de $\mathrm{F}$ nos dá uma idéia da força de explicação de um fator no desenho amostral escolhido(Tabela 1). Quão mais desproporcional for esta razão (p. ex., 2/144), maior a força de comparação entre os fatores estudados. Para ambos os estudos, observou-se grande força de comparação entre os fatores, o que auxilia na determinação do poder do teste estatístico, que é importante principalmente para monitorar impactos ambientais (6).

TABELA 1 - Desenho amostral para cada um dos estudos de caso (desenho 1 e 2). Todos fatores multiplicadores $(e, t, n)$ foram considerados fixos. Grau de liberdade (g.l.). Equação da soma dos quadrados e a razão pela qual será calculado o valor $F$

\begin{tabular}{|c|c|c|c|c|c|c|}
\hline \multirow{2}{*}{$\begin{array}{c}\text { Fatores } \\
\text { (desenho 1) }\end{array}$} & \multicolumn{3}{|c|}{ Multiplicadores } & \multirow{2}{*}{ g. 1.} & \multirow{2}{*}{$\begin{array}{l}\text { Soma dos } \\
\text { quadrados }\end{array}$} & \multirow{2}{*}{$\begin{array}{c}\text { Razão de F } \\
\text { (g. l. da razão) }\end{array}$} \\
\hline & e & $\mathbf{t}$ & $\mathbf{n}$ & & & \\
\hline $\mathrm{E}$ & 0 & $\mathrm{t}$ & $\mathrm{n}$ & 2 & $\delta^{2}+\mathrm{nt} \delta^{2} \mathrm{e}$ & Res (2/144) \\
\hline $\mathrm{T}$ & $\mathrm{e}$ & 0 & $\mathrm{n}$ & 11 & $\delta^{2}+$ ne $\delta^{2} \mathrm{t}$ & $\operatorname{Res}(11 / 144)$ \\
\hline $\mathrm{E} \times \mathrm{T}$ & 0 & 0 & $\mathrm{n}$ & 22 & $\delta^{2}+\mathrm{n} \delta^{2}$ et & $\operatorname{Res}(22 / 144)$ \\
\hline Residual & 1 & 1 & 1 & 144 & $\delta^{2}$ & \\
\hline Total & & & & 179 & & \\
\hline \multirow{2}{*}{$\begin{array}{c}\text { Fatores } \\
(\text { desenho 2) }\end{array}$} & \multicolumn{3}{|c|}{ Multiplicadores } & & Soma dos & Razão de F \\
\hline & c & t & $\mathbf{n}$ & g. 1. & quadrados & (g. l. da razão) \\
\hline $\mathrm{C}$ & 0 & $\mathrm{t}$ & $\mathrm{n}$ & 5 & $\delta^{2}+n t \delta^{2} \mathrm{c}$ & $\operatorname{Res}(5 / 144)$ \\
\hline $\mathrm{T}$ & $\mathrm{c}$ & 0 & $\mathrm{n}$ & 11 & $\delta^{2}+$ ne $\delta^{2} \mathrm{t}$ & $\operatorname{Res}(11 / 144)$ \\
\hline $\mathrm{C} \times \mathrm{T}$ & 0 & 0 & $\mathrm{n}$ & 55 & $\delta^{2}+\mathrm{n} \delta^{2} \mathrm{ct}$ & $\operatorname{Res}(55 / 144)$ \\
\hline Residual & 1 & 1 & 1 & 144 & $\delta^{2}$ & \\
\hline Total & & & & 215 & & \\
\hline
\end{tabular}


No entanto, embora exista grande potência em identificar os efeitos de cada fator ou da combinação destes, não se pode concluir que a variação observada foi devida exclusivamente a este(s) fator(es). O que temos é um exemplo de pseudo-replicação simples (7), onde o efeito observado não é necessariamente devido ao fator (p. ex.) energia ou contaminação e sim resultado da variação espacial inerente entre as praias estudadas. A pseudo-réplica é resultado do uso de estatística inferencial para testar efeitos de tratamentos de dados de experimentos cujos tratamentos não estão replicados ou cujas réplicas não são estatisticamente independentes (5). Ou seja, a pseudo-replicação não é um problema de planejamento amostral por si só (ou de amostragem) e sim uma combinação particular do desenho experimental (ou amostragem) e respectivas análises estatísticas que são inapropriadas para testar a hipótese de interesse.

Nos dois estudos de caso, o mesmo erro foi cometido. Embora o intuito fosse avaliar a influência da energia (Estudo 1) e da contaminação (Estudo 2), ambos os desenhos se restringiram somente às variações espaciais. Para realmente avaliar qualquer um dos fatores a que se propunham ambos os estudos, seriam necessárias réplicas praiais para cada condição, energética ou de contaminação, onde o efeito dos fatores pudesse ser avaliado em no mínimo três situações espaciais, comprovando, assim, que a variação encontrada entre as praias, dentro do grupo com o mesmo grau energético ou de contaminação, foi devida ao próprio fator e não a qualquer outro fator externo (8).

Outra constatação interessante é o elevado número de amostragens temporais que a maioria dos estudos ecológicos emprega. A amostragem em escala mensal só é válida e necessária se o evento que se pretende observar se modifica de mês para mês, tendo, assim, o tempo como um fator de análise, ou se pretende-se calcular, por exemplo, taxas de crescimento mensais de um determinado animal. Do contrário, a maioria dos eventos ecológicos em nível de comunidade varia de acordo com escalas temporais maiores, em nível sazonal e até anuais (8). Nestas condições, uma amostragem representativa em cada uma das estações do ano já é suficiente para constatar tais mudanças. Por estes motivos explanados, optou-se, neste novo desenho amostral (Figura 3), por aumentar as réplicas espaciais em detrimento das temporais.

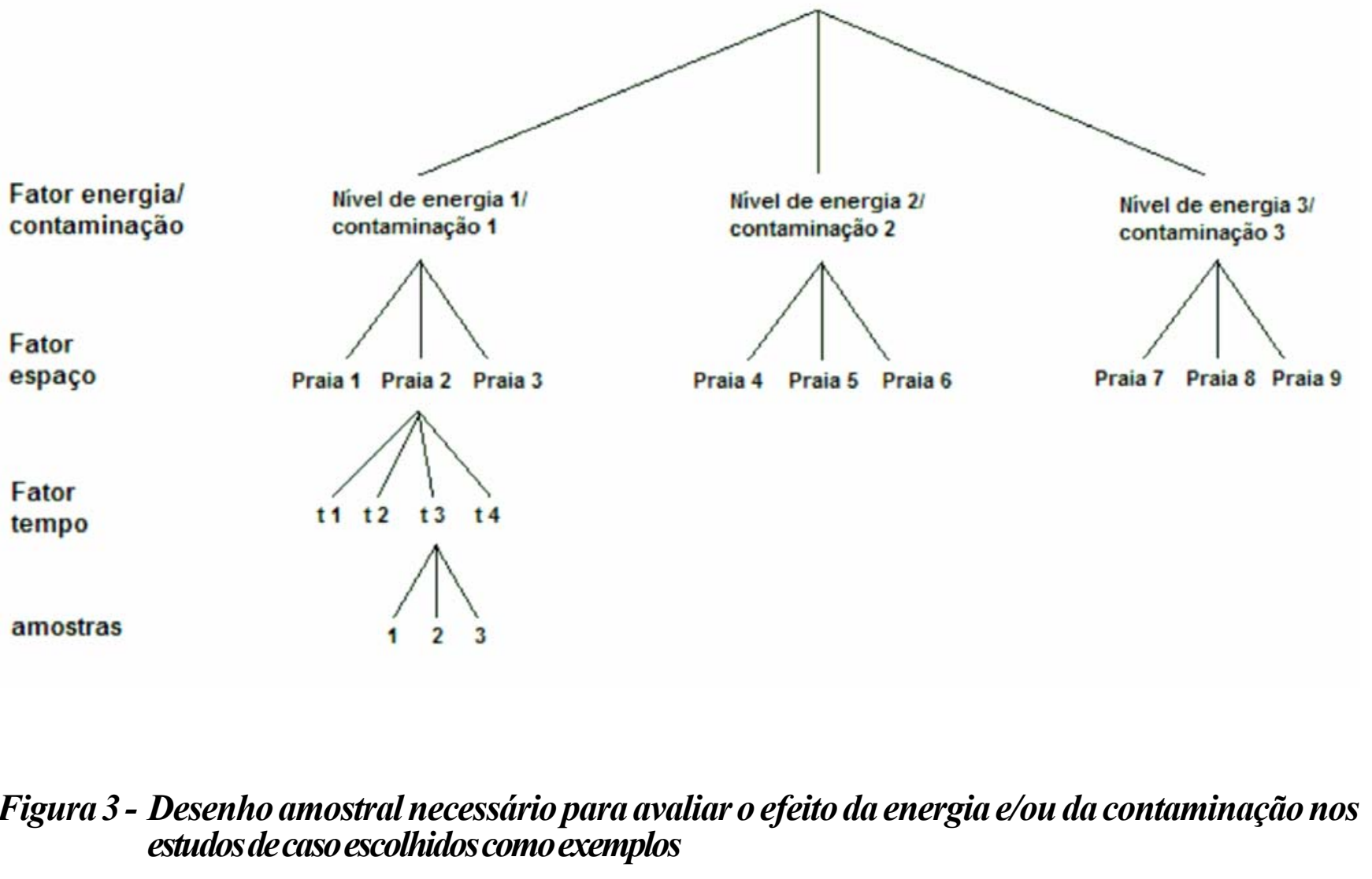
estudos de caso escolhidos como exemplos 
Conclusões

Há dois tipos principais de erros que se pode cometer em um estudo ecológico: o erro estatístico e o lógico. O primeiro é facilmente identificável e corrigido empregando-se ferramentas estatísticas apropriadas de acordo com os dados. Contudo, o segundo tipo de erro é de mais difícil percepção e pode levar a conclusões errôneas sobre as hipóteses levantadas, invalidando todo o estudo. Uma fonte comum deste tipo de erro são delineamentos amostrais pseudo-replicados, que levam a inferências lógicas erradas acerca da hipótese testada. Portanto, o desenho amostral é de fundamental importância para responder aos objetivos de um estudo, sendo sua resposta limitada principalmente pelo tipo de desenho empregado. Ao interpretar os resultados de uma análise estatística, deve-se ter em mente em que contexto amostral os dados foram obtidos e discuti-los com base em suas limitações.

\section{Referências}

1. Andrew NL, Mapstone BD. Samplign and the description of spatial pattern in marine ecology. Oceanography and Marine Biology Annual Review. 1987; 25:39-90.

2. Underwood AJ. Detection, interpretation, prediction and management of environmental disturbance: some roles for experimental marine ecology. Journal of Experimental Marine Biology and Ecology. 1996; 200:1-27.
3. Underwood AJ. Experiments in Ecology. their logical design and interpretation using analysis of variance. New York: Cambridge University Press, $\mathrm{UK} ; 1992$.

4. Green RH. Sampling design and statistical methods for environmental biologists. New York: Wiley, Chichester, UK; 1979. 272 p.

5. Hulbert SJ. Pseudoreplication and design of ecological field experiments. Ecological Monographs. 1984; 54:187-211.

6. Underwood AJ, Chapman MG. Power, precaution, Type II error and sampling desingn in assessment of environmental impacts. Journal of Experimental Marine Biology and Ecology. 2003; 296:49-70.

7. Krebs CJ. Ecological Methodology. New York: Harper and Row Publishers Inc; 1989. 654 p.

8. Underwood AJ. Experiments in ecology and management: Their logics, functions and interpretations. Australian Journal of Ecology. 1990; 15:365-389.

Recebido em/Received in: June 19, 2006 Aceito em/Accepted in: August 02, 2006 